\section{Interventional endoscopy}

\section{oxIV/1 721}

TRANESOPHAGEAL AND TRANSGASTRAL FINE NEEDLE ASPIRATION GUIDED BY ENDOSCOPIC ULTRASONOGRAPHY FOR THE DIAGNOSIS OF PARAGASTROESOPHAGEAL MASS LESIONS. M. Werener, R.J.. Adamek, B. Wedmann, B. Pfaffenbach. Dept of Medicine. St.Josef-Hospital, Ruhr-University Bochum, Germany

Endoscopic ultrasonography allows detailed study of the gastrointestinal wall and the surrounding tissues in case of paraesophageal mediastinal or paragastral space-occupying masses, however, cytologic confirmation is urgently required. which has been most commonly performed transcutaneously by CT guidance, but - as most echoendoscopes carry a biopsy puncture.

In 10 patients with paragastroesophageal mass lesions transesophageal or transgastralfine needle aspiratton (FNA) guided by endoscopic ultrasonography was performed using a convex 7.5 $\mathrm{MHz}$ transducer with longitudinal scanning in the axis of the endoscope (FG-32 UA, Pentax). In all patients conventional diagnostic techniques including transbronchial or esophageal "buttonhole" biopsies failed to obtain diagnostic tissue speciments.

Endosonographic guided FNA revealed bronchial carcinoma in 2 patients (one patient with upper vein blockage) (bronchoscopies negative), metastasis of mamma carcinoma compressing the esophagus in one patient (esophageal paragastral (intrathoracic stomach after esophagectomy for esophageal carcinoma) and paraesophageal after pneumectomy for bronchial carcinoma in 2 patients, adrenal gland adenoma in 2 patients and silicotic benign lymph node enlargement in one patient. In two patients with paraesophageal lymph node enlargement no definite diagnosis could be obtained. In all patients no complications were observed

Transesophageal and transgastral endosonographically guided FNA is an effective low-risk method for cytologic diagnosis of paragastro-esophageal ${ }^{-}$space-occupying mass lesions with difficult access by conventional techniques, particularty in the dorsal mediastinum. Endosongraphic convex transducers with longitudinal scanning are superior to those with radial scanning as the former enable the needle to be placed under continuous endosonographic visualization in the longitudinal axis of the echoendoscope.

\section{OXIV/3 1097}

ENDOSCOPIC TREATMENT OF BILE DUCT OBSTRUCTION AFTER ORTHOTOPIC LIVER TRANSPLANTATION.

A. Adler, W. Veltzke, R. Felix, P. Neuhaus, X.E. Hampel, R.E. Hintze. Dept of Gastroenterology, central interdiscipl. endoscopy, University Clinic Rudolf Virchow, Free University of Berlin, FRG.

Introduction: Up to $25 \%$ biliary complications ith cholestasis after orthotopic liver transplantation are described. This essentially depends on the anastomotic technique and can be reduced to 4.9 mosis (CdCdssa) (Tranisplant Prac 21: 2472(1989); Transplant.Proc 22: $15-71$ (1990)).

Patients and methods: In aur clinic from 9/88-11/92 $n=327$ orthotopic liver transplantations in $n=309$ pts. all with CdCdssa were performed. In this series we found in $n=9(2,8 \%)$ a relevant biliary duct obstruction. In all pts. We defined the bile duct obstruction by selective ERC and tried to remove the cholestasis by transpapillary techniques.

Results: Out of 9 we found the obstruction in 5 pts in the donors systen, in 3 in the cad pts. in the recipients system. In 3 pts. We had
multiple obstructions. Out of 5 pts. With findings multiple obstructions. Out of 5 pts. With findings
in donors system 3 had ischemia type biliary lesions, 1 had grafts rejection, I hemobilia with consecutive cholangitis following liver puncture. Within 3 pts. We had 1 with t-tube fragment impacted in the anastomosis, 2 pts. had stenosis of CdCdssa, 1 with leakage. 4 pts. With obstruction in the re cipients part of the biliary system had all papillary stenosis. First step of treatment was in $8 / 9$ pts. complete endoscopic sphincterotomy. In l case papillary dilatation was sufficient. Further endoscopic therapeutic procedures like basket-extraction and transpapillary drainage were necessary. In $6 / 9$ definitive removal of biliary obstruction was endoscopically performed. In $3 / 9$ intermittend biliary decompression was obtained. $2 / 9$ had to be reoperated. copy we could abolish biliary obstruction in $67 \%$ definify.
ENDOSCOPIC SPHINCTEROTOKY IS MORE HAZARDOUS IN PATIENTS UTTH SPHINCTER OF ODDI DYSFUNCTION AND NONDILATED BILE DUCTS. Y.K. Chen, M.J. Santoro, R.L. Follente, M.H. Halter, M.J. Collen. Division of Gastroenterology, Loma Linda University Medical Center, Loma Linda, CA, USA.

In the present study, we prospectively evaluated the relationship between common bile duct diameter. and the risk of sphincterotomy-Induced complications. Endoscopic retrograde cholangiopancreatography for sphincterotomy was done in 210 patients (62 males, 148 females), age $60 \pm 19$ years (meant1SD), who were divided into 3 groups: Group I - common duct stones, Group II - sphincter of OddI dysfunction, Group III - othe (5.2ళ) developed pancreatitis. Other sphincterotomy-induced complications were bleeding $(n-2)$, cholangitis $(n-2)$. retroperitoneal duodenal perforation $(n-1)$, and bile duct leakage $(n-1)$. 12 of the 210 patients were excluded from the analysis because of fallure to cannulate the common blle duct after precut sphincterotomy ( $n=2)$ or inadequate radiographs accurate measurement of common bile duct diameter $(n-10)$
TABLE I: sphincterotomy-induced pancreatitis and overali complication rates according to common bile duct diameter (chisquare analysis for trend)

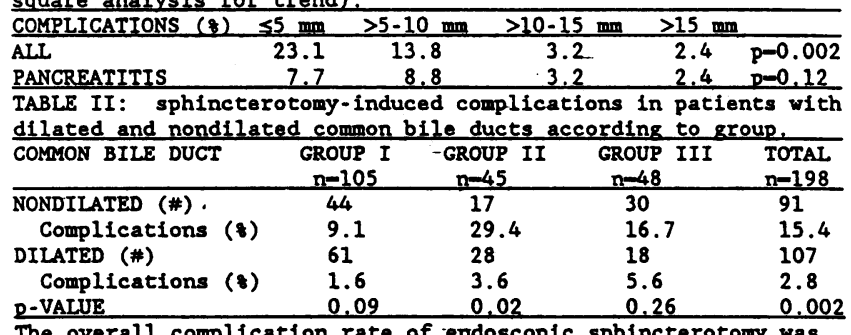

The overall complication rate of endoscopic sphincterotomy was 8.18: Group I - 4.68, Group II - 12.58, Group III - 11.18, $\mathrm{p}-0.16$. Pancreatitis occurred more frequently in Group II (12.5*) than in Group I (3.7ళ) or Group III (1.98), $p=0.03$. There was no significant difference in the complication rates between initial endoscopic sphincterotomy $(n-192)$ and repeat sphincterotomy $(n-18)(7.88$ vs $11.18, p-0.44)$. These results indicate that endoscopic sphincterotomy is more hazardous in patients. with sphincter of Oddi dysfunction and/or nondilated common bile ducts.

\section{OXIV/4 732}

INTERIM RESULTS OF THE NEW SELF-EXPANDABLE BILIARY PROSTHESIS (ENDOCOIL ${ }^{\text {Th }}$ ). E.Goldin, K. Huibregtse, T.Safra, M.Beyar, M.E.Smits, O.Globerman. Hadassah University
Hospital, Jerusalem, Israel and .Academic Medical Center, Aospital, Jerusalem, Israel
Amsterdam, The Netheriands.

We previously reported a new metallic stent, which successfully treated malignant obstructions in the biliary tract (Gastroint. Endosc. 1992, 38(2) 248). This selfexpandable stent (8mm diameter 4-8cm length) - a coil Inc.) - offers a strong radial force for very tight strictures, closed loops making tumor ingrowth very rare and soft ends preventing damage to the epithelium. Unti1 now, 25 of these stents were inserted in jaundice patients (15. in Jerusalem, 10 in Amsterdam) 13 females (52\%) and 12 males (48\%), with a median age of 74.1 (range 54-86). Lesions were pancreatic tumors in 23 patients and Cholangia $\mathrm{Ca}$ in 2. In 11 patients the stent was placed after clogging of plastic stents and in one patient inside an obstructed metallic. Wallstent. Stent implantation was technically successfur in all patients. However the stent delivery system was improved in the last cases to permit more -aecurate-location of the stent during deployment. In two patients the stent was removed because of failure of expansion, one of which developed bleeding and shock. A third patient had a severe pancreatitis soon after stent insertion and was operated upon. No other complications were noticed and relief of jaundice was achieved in all remaining patients. In three patients the stent was removed 2,4 and 5 months later respectively due to stent obstruction, by pulling the distal end of the stent, and new stents were inserted. Patients are continuing to be monitored for long term stent patency CONCLUSIONS: This new nickel-titanium stent is easily implanted and released, its strong radial force and coiled tubular form with tight loops permit good patency and very rare tumor ingrowth. The possibility of removing the stent is being improved and will permit its use also in benign strictures. The advantages of this stent are continuing to be evaluated. Randomized trials are necessary to compare the stent to the Wallstent and plastic stents. 
OXIV/5 1286

ENDOSCOPIC STENTING FOR OBSTRUCTIVE JAUNDICE IN PATIENTS WITH LIVER METASTASES FROM A DISTANT PRIMARY TUMOR K.E. Binmoeller. E. Goebell, N. Soehendra. Dept. of Endoscopic Surgery, Univ. Hospital, Hamburg, Germany

AIM: To evaluate the efficacy of endoscopic stenting for obstructive jaundice in pts with liver metastases from a primary malignancy originating outside of the region of the biliary tract ("distant" primary)

METHODS: Endoscopic retrograde cholangioscopy (ERC) records spanning a $10 \mathrm{yr}$ period $(9 / 82-9 / 92)$ were retrospectively reviewed. 59 patients ( 32 male, 27 female) with a mean age of 63 (range: 22-86) met the following criteria for inclusion in this study:

1. Liver metastases diagnosed by ultrasonography (US) and/or computed tomography (CT) and/or laparoscopy/lapararotomy 2. Dilated bile ducts on US and/or CT

3. Primary malignancy located outside of the region of the biliary tract.

Sites of the primary tumor were colon (37), breast (6), stomach (2), bronchial (2), and others (12). Mean serum bilinubin prior to ERC was $15 \mathrm{mg} \%$ (range:3.9-38).

RESULTS: ERC showed bile duct obstruction in-56 pts at the following levels: hilum (25 pts), mid-common duct ( 16 pts), distal common duct (15 pts). Stent placement (7-10 Fr) in these pts was successful in all but 1 pt. $75 \%$ of stented pts showed a reduction in serum bilirubin of at least $20 \%$ (mean: $56 \%$ ). Serum bilirubin normalized (<1 mg\%) in 9 pts ( $16 \%)$. Mean survival after stenting was 74 days (range: $4-299$ ). 19 pts had follow-up after hospital discharge; 13 reported an improvement of general wellbeing, 4 reported no change, and 2 reported symptomatic worsening.

CONCLUSIONS: Endoscopic stënting can provide effective palliation of obstructive jaundice in patients with liver metastases from a distant primary malignancy. However, the potential benefit of biliary decompression must be measured against a short survival period (mean of 74 days).

\section{OXIV/7 729}

COMBINED ENDOSCOPIC-LAPAROSCOPIC APPROACH IN PATIENTS WITH CHOLECYSTO-CHOLEDOCHOLITHIASIS. N. Basso, E. Fiocca, A. Materia, A. Fantini, G. Silecchia, E. Spaziani, A. Genco, C. Caruso, G. Pizzuto, A. Dé Leo. II Clinica Chirurgica, University "La Sapienza", Rome, Italy.

Laparoscopic Cholecystectomy (LC) combined with Endoscopic Sphincterotomy (EST) offers a chance of minimally invasive treatment of biliary stones.

Aim of this study was to review the results of this endoscopic laparoscopic approach. From November 1990 to January 1993613 consecutive patients with -symptomatic gallbladder disease were evaluated for $L C$

The preoperative work-up included: clinical history and physical examination, blood tests and ultrasonography (US). Endoscopic Retrograde Cholangio-Pancreatography (ERCP) was performed in case of: prevoius or present jaundice or acute pancreatitis; abnormal hepatic and/or pancreatic blood tests; CBD stones or dilated CBD $(>8 \mathrm{~mm}$ at US).

42-pts.-(6.8\%)-underwent-ERCP_because of: 17 previous jaundice (14) or acute pancreatitis (3); 5 hepatic and/or pancreatic altered blood tests; 11 CBD. stones and 9 dilated CBD at US. At ERCP 25 pts. (4.1\%) showed CBD stones and 9 a dilated CBD. 8 pts. showed a normal CBD at ERCP. EST was performed and stones extracted in 21 out of 25 pts. (84\%); of the remaining 4 pts.; 2 were treated by percutaneous transhepatic approach and 2 underwent laparotomy showing a cholecysto-choledocohal fistula. 2 out of 9 pts. with dilated CBD underwent EST because of papillary stenosis.

31 pts. (73.8\%) underwent LC 48 hours following EST. 9 out of 42 (21.4\%) presented with transient complications after ERCP/EST wich delayed LC (range 3-12 days). Considering the all series of 613 pts. CBD stones were preoperatively detected in $4.1 \%$ of cases.

This sequential therapeutic approach based on ERCP/EST plus LC is justified on a cost-benefit basis and succeeded in $84 \%$ of pts.

EST plus LC drammatically reduced the indications for laparotomy in pts. with cholecysto-choledocholithiasis.
ENDOSCOPIC STENTING IN THE MANAGEMENT OF BILIARY STONES

NM LAW, CC LIM, HS NG, J CHENG

DEPARTMENT OF MEDICINE $I$

SINGAPORE GENERAL HOSPITAL, SINGAPORE

Endoscopic Retrograde Cholangiopancreatography (ERCP) is now an established mode of treatment in the management of bile duct stones and cholangitis. This is important when the patients are elderly, and have other medical conditions rendering them unfit for surgery.

We studied the long term outcome of endoscopic stenting in patients who underwent ERCP for ductal stones. From January 1990 to April 1992, we performed 894 ERCP's in the Department, of which, 366 were for biliary stone disease. Of these, 55 patients received a-10 French biliary stent for the treatment of bile duct stones All the patients bad sphincterotomy while Dormia basket the 13 patients. Most of these patients $(60 \%)$ had an advanced age of more than 70 years. Eighty two percent had multiple stones. Ninety one percent had stones greater than $1.2 \mathrm{~cm}$ in diameter. The patients were followed up for a mean period of 11 months. Eighty two percent of patients had relief of jaundice and cholangitis subsided in $95 \%$. Nineteen patients still have their stents in place and no surgery was required. Nineteen patients went for surgery at a later date when they became more stable. None required immediate surgery post ERCP. Eight patients died of unrelated illnesses and 4 defaulted follow up.

The stents were repeatedly changed in 15 patients after an average duration of 5.5 months. In 5 patients, the stones have either disappeared spontaneously or become smaller and removed at subsequent ERCP. Complications included cholangitis in 2 patients and stent migration in one. There was no mortality.

In conclusion, stenting is a valuable alternative to surgical bile duct exploration and in some cases may be the definitive mode of treatment for large or multiple bile duct stones.
EFFICIENCY OF TRANSPAPILLARY ENDOSCOPIC TREATMENT OF THE BILIARY TRACT IN PATIENTS WITH BILLROTH-II GASTRIC RESECTION:

R.E. Hintze, A. Adler, W. Veltzke, K:E. Hanpel. Dept. of R.E. Hintze, A. Adler, W. Veltzke, K.:. hampel. Dept. of Clinic Rudolf Virchow, Free University of Berlin, FRG.

Introduction: In Billroth-II-patients problems in papillary endoscopy result from 1 . the difficulties of the access to the duodenum, 2. the upside-down position of the endoscope in front of the papilla and 3. hereby following special regard to cannulation and sphincterotomy. Concerning to these reain comparision to the normal anatomy. In order to improve the results we developed a Billroth-IIadapted Erlangen type papillotome. We are reporting the results of our study using this instrument. Patients: From 1/83-10/92 we found in $n=3688$ pts. with indication for endoscopic sphincterotomy $n=$ $125(3,4 \%)$ with Billroth-II-anatomy. Brauns entero anastomosis was in $68 \%$ of the pts., in $28 \%$ there was none, and in $4 \%$ there was a Roux-Y-interposition Results: In 111 of 125 pts. $(88,8 \%)$ we were able to perform complete sphincterotomy and to abolish definitely biliary obstruction by different methods (gall stone extraction, lithotripsie and transpapir lary drainage) depending on the indication. The re son papilla, three of them with a Roux-Y-interposition. Regarding the pts. With classical Billroth-II-situs twe were in 109 out of $120(90,8 \%)$ successfull. Due to the increasing experience with this method in a last series from $8 / 90-10 / 92(n=29)$ was a successrate of $96 \%$. There were no complications.

Conclusions. The efficiency of transpapillary endos copic treatment in bile duct obstruction of Billroth- using our special adapted Erlangen-type papillotome is with $96 \%$ similar to that of nongastric resected patients. Roux-Y-interposition re duces success-rate to $60 \%$. In nearly all cases we could by endoscopic procedures prevent biliary surgery. 